University of South Carolina

Scholar Commons

$11-25-2002$

\title{
Polarization Effects in Photoluminescence of C- and M-Plane GaN/AIGaN Multiple Quantum Wells
}

\author{
E. Kuokstis \\ C. Q. Chen \\ M. E. Gaevski \\ W. H. Sun \\ J. W. Yang
}

See next page for additional authors

Follow this and additional works at: https://scholarcommons.sc.edu/elct_facpub

Part of the Electromagnetics and Photonics Commons, and the Other Electrical and Computer

Engineering Commons

\section{Publication Info}

Published in Applied Physics Letters, Volume 81, Issue 22, 2002, pages 4130-4132.

(C)Applied Physics Letters 2002, American Institute of Physics (AIP).

Kuokstis, E., Chen, C. Q., Gaevski, M. E., Sun, W. H., Yang, J. W., Simin, G., Khan, N. A., Maruska, H. P., Hill, D. W., Chou, M. C., Gallagher, J. J., \& Chai, B. (25 November 2002). Polarization Effects in Photoluminescence of C- and M-Plane GaN/AIGaN Multiple Quantum Wells. Applied Physics Letters, 81 (22), 4130-4132. http://dx.doi.org/10.1063/1.1524298

This Article is brought to you by the Electrical Engineering, Department of at Scholar Commons. It has been accepted for inclusion in Faculty Publications by an authorized administrator of Scholar Commons. For more information, please contact digres@mailbox.sc.edu. 


\section{Author(s)}

E. Kuokstis, C. Q. Chen, M. E. Gaevski, W. H. Sun, J. W. Yang, Grigory Simin, M. Asif Khan, H. P. Maruska, D. W. Hill, M. C. Chou, J. J. Gallagher, and B. Chai 


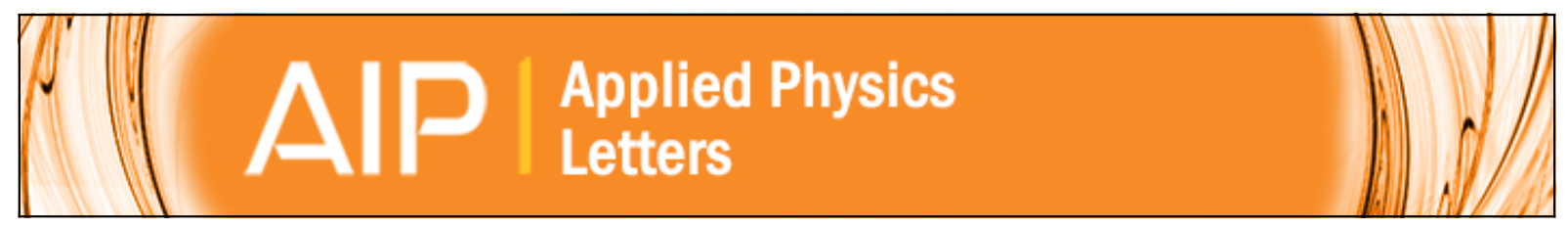

\section{Polarization effects in photoluminescence of C - and M -plane GaN/AIGaN multiple quantum wells}

E. Kuokstis, C. Q. Chen, M. E. Gaevski, W. H. Sun, J. W. Yang, G. Simin, M. Asif Khan, H. P. Maruska, D. W. Hill, M. C. Chou, J. J. Gallagher, and B. Chai

Citation: Applied Physics Letters 81, 4130 (2002); doi: 10.1063/1.1524298

View online: http://dx.doi.org/10.1063/1.1524298

View Table of Contents: http://scitation.aip.org/content/aip/journal/apl/81/22?ver=pdfcov

Published by the AIP Publishing

\section{Articles you may be interested in}

Optical polarization anisotropy of a -plane GaN/AIGaN multiple quantum well structures grown on $r$-plane sapphire substrates

J. Appl. Phys. 105, 123112 (2009); 10.1063/1.3156688

Well-width dependence of photoluminescence emission from a-plane GaN/AIGaN multiple quantum wells Appl. Phys. Lett. 84, 496 (2004); 10.1063/1.1638884

Photoluminescence of InGaN/GaN multiple quantum wells originating from complete phase separation J. Appl. Phys. 93, 2507 (2003); 10.1063/1.1540738

Localization of carriers and polarization effects in quaternary AllnGaN multiple quantum wells Appl. Phys. Lett. 79, 4375 (2001); 10.1063/1.1429753

In-plane polarization anisotropy of the spontaneous emission of M-plane $\mathrm{GaN} /(\mathrm{Al}, \mathrm{Ga}) \mathrm{N}$ quantum wells Appl. Phys. Lett. 77, 3343 (2000); 10.1063/1.1326846

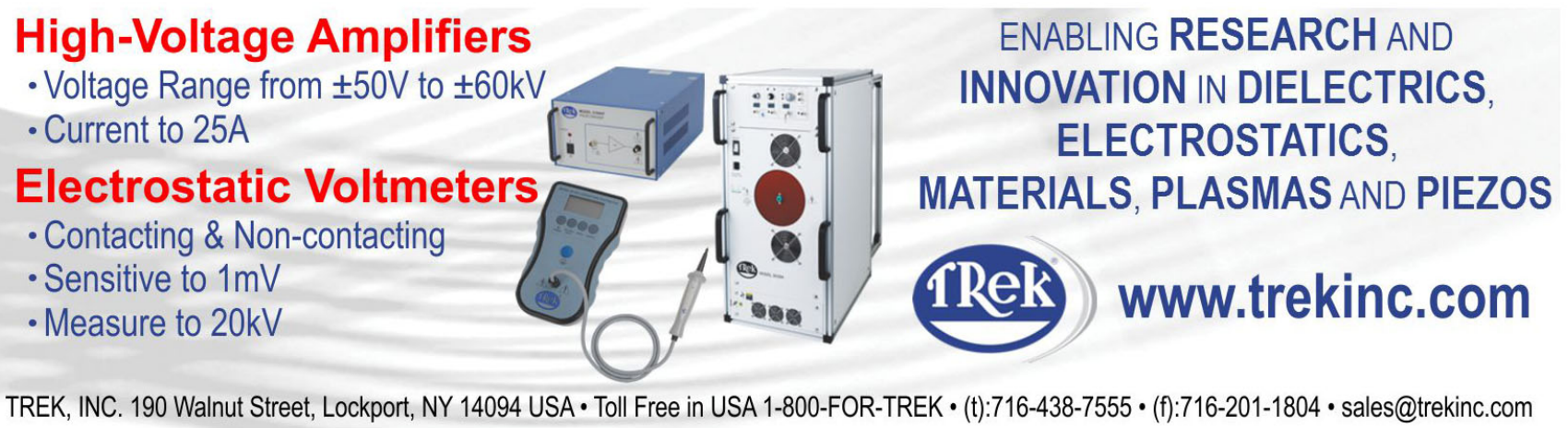




\title{
Polarization effects in photoluminescence of $C$ - and $M$-plane GaN/AIGaN multiple quantum wells
}

\author{
E. Kuokstis, ${ }^{\text {a) }}$ C. Q. Chen, M. E. Gaevski, W. H. Sun, J. W. Yang, G. Simin, \\ and M. Asif Khan \\ Department of Electrical Engineering, University of South Carolina, 301 South Main Street, \\ Columbia, South Carolina 29208
}

H. P. Maruska, D. W. Hill, M. C. Chou, J. J. Gallagher, and B. Chai Crystal Photonics, Incorporated, Sanford, Florida 32773

(Received 23 July 2002; accepted 2 October 2002)

\begin{abstract}
Polarization effects have been studied in GaN/AlGaN multiple quantum wells (MQWs) with different $c$-axis orientation by means of excitation-dependent photoluminescence (PL) analysis. Quantum structures were grown on [0001]-oriented sapphire substrates ( $C$ plane) and single-crystalline [1 100$]$-oriented freestanding $\mathrm{GaN}$ ( $M$ plane) using the metalorganic chemical vapor deposition technique. Strong PL spectrum line blueshifts (up to $140 \mathrm{meV}$ ) which are correlated with the excitation intensity have been obtained for $C$-plane MQWs, whereas no shift has been observed for $M$-plane MQWs. Theoretical calculations and comparison with the PL data confirm that the built-in electric field for $C$-plane structures is much stronger than the field present for $M$-plane MQWs. In the former case, the excitation-induced blueshift of the PL line is due to the screening of the built-in electric field by photoinjected carriers, which is consistent with the field strength of $1.23 \mathrm{MV} / \mathrm{cm}$ in the absence of excitation. (C) 2002 American Institute of Physics.
\end{abstract}

[DOI: $10.1063 / 1.1524298]$

The III-nitride semiconductors and their alloys are attractive materials suitable for the development of blue and UV optoelectronic devices (light-emitting and laser diodes, photodetectors), as well as for high-voltage and high-power electronic devices (e.g. high-electron mobility transistors). ${ }^{1,2}$ Various epilayers, including quantum structures such as single or multiple quantum wells (MQWs) are most commonly grown on [0001]-oriented sapphire or 6H-SiC. With this orientation, strong built-in electrostatic fields appear as a result of spontaneous and piezoelectric polarization, ${ }^{3}$ due to the noncentrosymmetric nature of wurtzite $C$-plane oriented films. These electrostatic fields tend to bend the energy band structure of quantum wells, and electrons and holes become situated in nearly triangular potential wells. The modulation of the conduction and valence band edges due to large internal electric fields leads to the quantum confined stark effect (QCSE). The QCSE is undesirable in light-emitting devices because the fields across the individual quantum wells give rise to a spatial separation of electrons and holes. ${ }^{4}$ This results in a reduced oscillator strength and lower quantum efficiency for radiative transitions. Furthermore, in MQW structures designed for deep UV emission, QCSE causes an undesirable redshift in the emission spectra. ${ }^{5}$

A useful approach for reducing the deleterious effects of built-in fields is to fabricate GaN-based structures along nonpolar directions, e.g., to grow [1 $\overline{100}]$-oriented $M$-plane films since these surfaces contain an equal number of $\mathrm{Ga}$ and $\mathrm{N}$ atoms and, therefore, are nonpolar. ${ }^{6}$

In this letter, we study processes in highly excited GaN/ AlGaN MQWs with $C$ - and $M$-plane surfaces and, thereby,

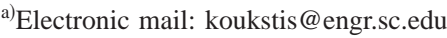

we seek to analyze the effects of controlling a built-in electrostatic field in determining the properties of quantum structures. Six periods of the $\mathrm{AlGaN} / \mathrm{GaN}$ quantum wells were simultaneously deposited on the freestanding $M$-plane $\mathrm{GaN}$ wafers and thick $C$-plane $\mathrm{GaN}$ layers under identical conditions. The [1 $\overline{100}]$-oriented $M$-plane $\mathrm{GaN}$ templates were grown by halide phase epitaxy on the closely lattice matched (100) plane of $\mathrm{LiAlO}_{2}$. After depositing a 350- $\mu$ m-thick layer of $\mathrm{GaN}$, the original $\mathrm{LiAlO}_{2}$ substrate was removed with wet acid etching. Thus, freestanding [1 100$]$-oriented $\mathrm{GaN}$ templates were obtained, which served as the starting substrates for our study. The $2 \theta-\omega \mathrm{x}$-ray diffraction (XRD) scans exhibit only sharp GaN (1 $\overline{100})$ and (2 200$)$ peaks, indicating the single-crystal nature of (1 $\overline{100})$ plane GaN templates. The $\omega$ scans show a full width at half maximum of about 20 arcmin indicating reasonable crystal quality. The [0001]-oriented $C$-plane 3- $\mu \mathrm{m}$-thick layers were grown on sapphire. The AlGaN/GaN MQWs growth was carried out by low-pressure metalorganic chemical vapor deposition. Triethylgallium, triethylaluminum (TEA), and $\mathrm{NH}_{3}$ were used as the precursors for $\mathrm{Ga}, \mathrm{Al}$, and $\mathrm{N}$, respectively. TEA has been chosen as the Al source because of its low vapor pressure. Thus, we are able to deposit the AlGaN quantum well barrier films with an appropriately slow growth rate to obtain a sharp interface. The reactor pressure and temperature were kept at 76 Torr and $1000{ }^{\circ} \mathrm{C}$ and $\mathrm{H}_{2}$ was used as a carrier gas during the growth. From high-resolution XRD scan curves, we evaluated the MQW well width and barrier width $L_{w}=5$ $\mathrm{nm}$ and $L_{b}=10 \mathrm{~nm}$, respectively. These values agree very well with the thickness values based on growth rate. The smooth surface of the samples was revealed by a Nomarsky optical microscopy and scanning electron microscopy. The barrier alloy contained $18 \%$ of $\mathrm{Al}$. The photoluminescence 


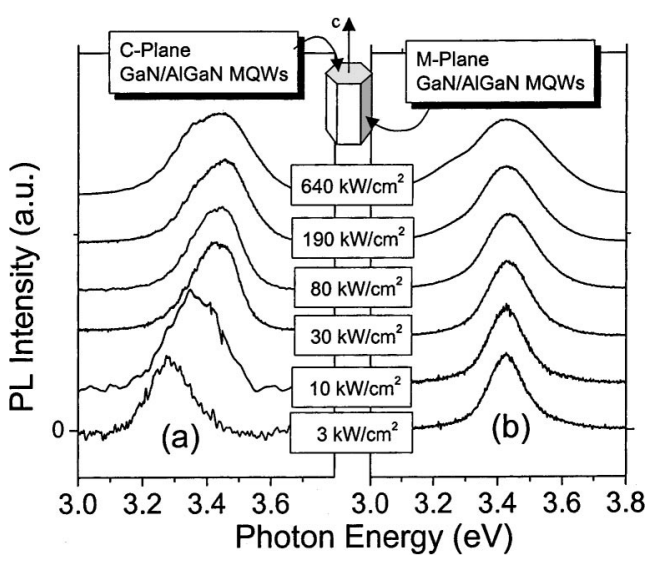

FIG. 1. RT PL spectra for $C$-plane (a) and $M$-plane (b) $\mathrm{GaN} / \mathrm{Al}_{0.18} \mathrm{Ga}_{0.82} \mathrm{~N}$ MQWs as a function of ArF excimer laser excitation power density. The inset shows wurtzite structure film surface orientation.

(PL) spectra were then measured using pulsed $\operatorname{ArF}$ excimer laser excitation $\left(\lambda=193 \mathrm{~nm}, \tau=8 \mathrm{~ns}\right.$, and $\left.f_{\text {rep }}=10-100 \mathrm{~Hz}\right)$. The laser beam was focused on the surface of the samples to a spot of about $0.2 \mathrm{~mm}$ diameter. A maximum pump power density of $\sim 2 \mathrm{MW} / \mathrm{cm}^{2}$ could thus be reached. Excitation intensity could be reduced by a set of neutral density filters. Luminescence was measured in a backscattering geometry using a SPEX550 monochromator with a UV-enhanced charge coupled device array.

In this study, we concentrate our attention on the roomtemperature (RT) experiments, since at low-temperature, PL analysis is more complicated due to spectral structures of exciton origin (free, bound excitons, their phonon replicas) and competition of these recombination channels. Figure 1 shows RT PL spectra for $C$-plane [Fig. 1(a)] and $M$-plane [Fig. 1(b)] of $\mathrm{GaN} / \mathrm{Al}_{0.18} \mathrm{Ga}_{0.82} \mathrm{~N}$ MQWs under conditions of different excitation power density. In both cases, the near band-edge emission spectrum consists of one band; however, for the $C$-plane sample, the peak undergoes a blueshift with excitation intensity; whereas for the $M$-plane, its position remains unchanged. The long-wave shoulder of the spectra which appears under highest excitation $\left(>500 \mathrm{~kW} / \mathrm{cm}^{2}\right)$ may be ascribed to many-body effects in high-density electronhole plasma. ${ }^{7}$ The experimental points in Fig. 2 show the peak energies of the PL spectra as a function of excitation



FIG. 2. Dependence of peak energy of the PL spectrum on excitation power density. Symbols correspond to experimental data and solid curves correspond to theoretical calculations. Curves 1,2, and 3 were calculated using constant lifetime approach, and curves 4, 5, and 6 were obtained using bimolecular recombination model. Curves 1 and 4 correspond to built-in electric field strength $F_{0}=1.23 \mathrm{MV} / \mathrm{cm}$, curves 2 and 5 correspond to $F_{0}=0.75 \mathrm{MV} / \mathrm{cm}$, and curves 3 and 6 correspond to $F_{0}=0.3 \mathrm{MV} / \mathrm{cm}$.
TABLE I. Material parameters used in calculations. For $\mathrm{Al}_{0.18} \mathrm{Ga}_{0.82} \mathrm{~N}$, linear extrapolation was applied.

\begin{tabular}{lcc}
\hline \hline Parameter & \multicolumn{1}{c}{$\mathrm{GaN}$} & $\mathrm{Al}_{0.18} \mathrm{Ga}_{0.82} \mathrm{~N}$ \\
\hline$E_{g}(\mathrm{eV})$ & 3.4 & 3.9 \\
$m_{e}^{*}\left(m_{0}\right)$ & 0.2 & \\
$m_{h}^{*}\left(m_{0}\right)$ & $\sim 1$ & \\
$\alpha\left(\mathrm{cm}^{-1}\right)$ & $3 \times 10^{5}$ & \\
$\tau_{r}(\mathrm{~ns})$ & $\sim 1$ & \\
$\gamma\left(\mathrm{cm}^{3} / \mathrm{s}\right)$ & $2.4 \times 10^{-11}$ & \\
$\epsilon_{r}(\mathrm{relative})$ & 9.5 & \\
$a(\AA)$ & 3.189 & 3.175 \\
$\sigma_{\|}(\%)$ & 0 & 0.439 \\
$e_{31}\left(\mathrm{C} / \mathrm{m}^{2}\right)$ & -0.49 & -0.51 \\
$e_{33}\left(\mathrm{C} / \mathrm{m}^{2}\right)$ & 0.73 & 0.86 \\
$C_{13} \times 10^{11}\left(\mathrm{dyn} / \mathrm{cm}^{2}\right)$ & 10.8 & 11.0 \\
$C_{33} \times 10^{11}\left(\mathrm{dyn} / \mathrm{cm}^{2}\right)$ & 39.9 & 39.8 \\
$P_{\text {sp }}\left(\mathrm{C} / \mathrm{m}^{2}\right)$ & -0.029 & -0.0348 \\
\hline \hline
\end{tabular}

power density $P$ with more detail. Note that for $P$ $>50 \mathrm{~kW} / \mathrm{cm}^{2}$, PL spectral positions, in both cases, become very close and remain nearly the same with further increases in excitation intensity. The observed PL dependencies may be explained taking into account the built-in electrostatic field, the strength of which reduces with increasing excitation due to field screening by the carriers. In order to evaluate the magnitude of the electric field in the MQWs, we applied a simple model based on a triangular potential well resulting from the presence of both the total built-in field as well as the photogenerated carriers with two-dimensional (2D) carrier density $\sigma$. We assumed that those carriers accumulate at GaN/AlGaN interfaces, thereby reducing the field $F_{w}$, which can be approximately evaluated as ${ }^{8}$

$$
F_{w}(\sigma)=F_{0}-\sigma e L_{b} /\left[\epsilon_{0}\left(L_{w} \epsilon_{b}+L_{b} \epsilon_{w}\right)\right] .
$$

Here, $F_{0}$ is built-in electrostatic field strength in unexcited wells. All the parameters used in our calculations are taken from literature ${ }^{3,9-11}$ and are listed in Table I. Since the position of the PL peak energy $h \nu_{\max }$ for near band-edge emission (electron-hole band-to-band and/or excitonic recombination) is determined by the ground level energy, we can obtain $^{12}$

$$
\begin{aligned}
h \nu_{\max }= & E_{g}-e F_{w}(\sigma) L_{w}+\left[\frac{9 e h F_{w}(\sigma)}{16 \sqrt{2}}\right]^{2 / 3}\left[\left(\frac{1}{m_{e}^{*}}\right)^{1 / 3}\right. \\
& \left.+\left(\frac{1}{m_{h}^{*}}\right)^{1 / 3}\right] .
\end{aligned}
$$

In order to compare our experimental results with calculations, we have to express $h \nu_{\max }$ as a function of excitation power density $P$. We have used two approaches: (i) assuming constant carrier lifetime $\tau_{r}$ (this situation is expected for lower carrier injection level) or (ii) assuming predominantly bimolecular recombination (higher excitation). In the former case, the $2 \mathrm{D}$ carrier density $\sigma=\alpha P \tau L_{w} / h \nu_{\text {las }}$, whereas in the latter case $\sigma=L_{w} \sqrt{P \alpha / h \nu_{\text {las }} \gamma}$. Here $\alpha$ is the absorption coefficient for the laser emission at $h \nu_{\text {las }}$ and $\gamma$ is the bimolecular recombination coefficient (see Table I). The results of calculations for several electric field values are shown in Fig. 2. It can be seen that the best fit with experimental data for $C$-plane MOWs was obtained for $F_{0}=1.23 \mathrm{MV} / \mathrm{cm}$ (curves 1 


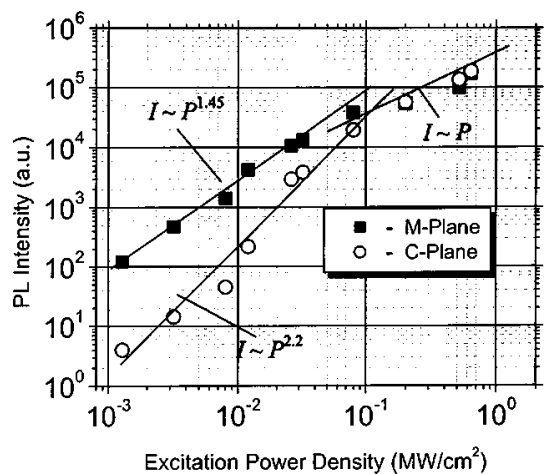

FIG. 3. PL intensity dependence on excitation power density for $C$-plane (filled squares) and $M$-plane (open circles) GaN/AlGaN MQWs. Solid lines represent different power dependences $I \sim P^{\beta}$ ( $\beta=1,1.45$, and 2.2).

and 4 in Fig. 2), whereas for $M$-plane MQWs, the initial field values need to be much lower $(<300 \mathrm{kV} / \mathrm{cm}$, curves 3 and $6)$. From this comparison, we can also conclude that the constant lifetime approach describes experiment results better for lower excitation levels (curve 1), while bimolecular recombination presumably starts to appear at $P>10-20$ $\mathrm{kW} / \mathrm{cm}^{2}$ (curve 4).

We have evaluated the built-in electric field $F_{0}$ in the case of spontaneous and piezoelectric polarization in $\mathrm{GaN} / \mathrm{Al}_{0.18} \mathrm{Ga}_{0.82} \mathrm{~N}$ MQWs ( $C$-plane orientation) independently, just taking into account the parameters relevant to those quantum structures. Indeed, using parameters from Table I, we can obtain ${ }^{8}$

$$
F_{0}=\left(P_{s p}^{w}-P_{s p}^{b}-P_{p e}^{b}\right) L_{b} /\left[\epsilon_{0}\left(L_{w} \epsilon_{b}+L_{b} \epsilon_{w}\right)\right] .
$$

In this model, we have neglected piezopolarization in the wells since the well material is GaN and suffers no in-plane deformation due to the thick (therefore, relaxed) GaN buffer layer of the structures. However, the $\mathrm{Al}_{0.18} \mathrm{Ga}_{0.82} \mathrm{~N}$ barrier is under the tension $\sigma_{\|}$due to in-plane mismatch with $\mathrm{GaN}$ and, hence, barrier piezoelectric charge can be calculated as $P_{\mathrm{pe}}^{b}=-2 \sigma_{\|}\left(e_{33} C_{11} / C_{33}-e_{31}\right)$, where $e_{i j}$ and $C_{i j}$ are piezoelectric tensor components and the elastic constants, respectively (see Table I). The field value evaluated according to Eq. (3) for $C$-plane MQWs is found to be $1.27 \mathrm{MV} / \mathrm{cm}$. It agrees well with the value $1.23 \mathrm{MV} / \mathrm{cm}$ obtained from our earlier theoretical and experimental PL data fitting in Fig. 2.

In addition, we have analyzed the PL excitation intensity dependence of the PL for both $M$ - and $C$-plane MQWs over a wide range of intensities. Figure 3 shows integrated PL intensity as a function of excitation power density. Note that under higher excitation levels, $\left(P>50 \mathrm{~kW} / \mathrm{cm}^{2}\right)$ absolute PL intensity is very close for both $C$ - and $M$-plane MQWs and increases linearly with excitation $(I \sim P)$; whereas at low excitation levels, the situation is quite different. At low levels, the PL intensity in $C$-plane MQWs increases strongly superlinearly $\left(I \sim P^{2.2}\right)$; whereas in $M$-plane MQWs, the dependence is weaker $\left(I \sim P^{1.45}\right)$. At the excitation level of $\sim 1$ $\mathrm{kW} / \mathrm{cm}^{2}$, the PL intensity from MQWs with $M$-plane orientation is about 30 times higher in comparison to those with $C$-plane orientation. A similar difference in PL intensities was reported recently in quantum well structures with $A$-plane orientation using continuous wave laser excitation. ${ }^{13}$ These observations can be understood by taking into account the strong built-in electric field in $C$-plane MQWs in contrast to $M$-plane MQWs which exhibit much lower field strength. Indeed, spatial separation of carriers in triangular wells due to the QCSE in $C$-plane MQWs with the characteristic strong built-in fields which exist at low excitation intensities leads to poor overlapping of the wave functions of electrons and holes. This process leads to the major reduction of electronhole radiative recombination traffic as compared to $M$-plane MQWs under these conditions. Typically, when nonradiative channels predominate (low excitation and constant lifetime regime), the band-to-band or excitonic radiative recombination rate is expected to change with excitation as $I \sim P^{2}$. There will be a transformation to $I \sim P$ when nonradiative channels saturate and radiative recombination predominates at elevated excitation (high excitation and bimolecular recombination regime). A power index 2.2 for $C$-plane MQWs indicates that there must be some additional reason for a steeper PL intensity dependence on excitation. We suggest that the reason is based on screening of the built-in electric field by injected carriers at elevated excitation levels, whereby the reduction of the field strength leads to a stronger overlap of the carrier wave functions and, consequently, an additional increase of PL intensity.

In conclusion, the comparative study of PL for $C$ - and $M$-plane GaN/AlGaN MQWs showed strong differences in excitation-induced behavior of PL in these quantum structures. We found that for $C$-plane oriented MQWs, the energy of the peak of the PL spectrum became blueshifted with increasing excitation intensity, whereas the peak position of the PL for $M$-plane MQWs remained unchanged. We observed much weaker PL intensity for $C$-plane MQWs in comparison to $M$-plane MQWs at low excitation levels. These results indicate the existence of much stronger built-in electric fields in $C$-plane quantum structures in comparison with $M$-plane MQWs. An analysis of the experimental results along with theoretical data fitting shows that the electric-field strength in $C$-plane MQWs reaches the value of $1.23 \mathrm{MV} / \mathrm{cm}$. Clearly, the growth of GaN/AlGaN MQWs with a nonpolar orientation can lead to a significant increase in the quantum efficiency of these nitride-based emitters, especially at low and medium excitation levels (e.g., for light-emitting diodes).

This work at USC was supported by Army SMDC Contract No. DASG60-00-10003, monitored by Terry Bauer.

${ }^{1}$ S. Nakamura and G. Fasol, The Blue Laser Diode (Springer, Heidelberg, 1997).

${ }^{2}$ S. J. Pearton, J. C. Zolper, R. J. Shul, and F. Ren, J. Appl. Phys. 86, 1 (1999).

${ }^{3}$ F. Bernardini, V. Fiorentini, and D. Vanderbilt, Phys. Rev. B 56, R10024 (1997).

${ }^{4}$ J. S. Im, H. Kollmer, J. Off, A. Sohmer, F. Scholz, and A. Hangleiter, Phys. Rev. B 57, R9435 (1998)

${ }^{5}$ G. Vaschenko, D. Patel, C. S. Menoni, N. F. Gardner, J. Sun, W. Götz, C. N. Toné, and B. Clausen, Phys. Rev. B 64, 241308 (2001).

${ }^{6}$ J. E. Northrup and J. Neugebauer, Phys. Rev. B 53, R10477 (1996).

${ }^{7}$ H. Haug and S. W. Koch, Phys. Rev. A 39, 1887 (1989).

${ }^{8}$ R. Cingolani, A. Botchkarev, H. Tang, and H. Markoç, Phys. Rev. B 61, 2711 (2000).

${ }^{9}$ D. W. Palmer, www.semiconductors.co.uk, 2001.04.

${ }^{10}$ S. C. Jain, M. Willander, J. Narayan, and R. Van Overstraeten, J. Appl. Phys. 87, 965 (2000).

${ }^{11}$ A. Dmitriev and A. Oruzheinikov, J. Appl. Phys. 86, 3241 (1999).

${ }^{12}$ C. Weisbuch and B. Vinter, Quantum Semiconductor Structures (Academic, New York, 1991)

${ }^{13}$ H. M. Ng, Appl. Phys. Lett. 80, 4369 (2002). 